\title{
Behavior management in Pediatric Dentistry during and after Corona pandemic
}

\author{
Sonu Acharya ${ }^{1}-$
}

\section{Highlights}

The current paper mainly focuses on how the things have changed and what are the specific changes that we can incorporate in our clinics so that we can make it less fearful to the children in present scenario.

1 Professor at Department of Pediatric and Preventive Dentistry, Institute of Dental sciences, $S O A$ (Deemed to be University), Bhubaneswar, Odisha, India-751003

\section{Correspondence:}

Institute of Dental sciences, SOA(Deemed to be University), Bhubaneswar, Odisha, India-751003 E-mail address:

sonu ain@yahoo.com
The present pandemic situation has brought about significant changes within the dental clinics and their environment. Each and everything had undergone transformation that has become a "new normal".
Pediatric dentists must adopt all the required behavior management skills and be cautious about the whole procedure.

\section{Abstract}

Children are not miniature adults. They should be treated as different entity and not just as adults who are shortened. Children have their own apprehensions and fear. The most commonly encountered is the fear of the unknown. Fear is something which is innate and inborn. Fear of dentistry is there in every individual, whether young or old. This is mostly subjective in nature. Children show exaggerated fear for dentistry as this is something new to them. The pediatric dentists are trained in behavior management for reducing fear. There have been many methods, both pharmacological and nonpharmacological for behavior management in children. The pediatric dentists were able to apply one or the other behavior management techniques till now. Sudden appearance of a pandemic caused by nCOV-2 (coronavirus) changed everything. The change in practice pattern is bound to effect all the dental patients, more so the children. Here we will discuss how the behavior management techniques will change for children and how we can get some newer methods to reduce the fear.

Keywords: Behavior Management; Coronavirus; COVID-19; Pediatric Dentistry 


\section{INTRODUCTION}

Pediatric dentists are expected to identify and effectively treat dental diseases of children that are within the knowledge and scope acquired during their professional studies. Effective and efficient treatment has to be provided to the child and for that, the parents also have to understand their roles. ${ }^{1}$ Behavior guidance should be done and this means that inappropriate behavior has to be converted to appropriate behavior within the societal norms with the help of empathy and learning. ${ }^{2}$ The main idea behind behavior management by pediatric dentist is to gain the confidence of the parents and the child. It's always better to know the scientific basis of implementation of any behavior management technique and also to look into cultural sensitivities, tolerance level and flexibility in a child, and the parents before applying any of these methods. ${ }^{3}$ Children are not miniature adults. They should be treated as different entity and must be dealt with carefully. Children have their own apprehensions and fear. Behavior management should never be used as a punishment or assertiveness for a child. All the methods which are to be used to tame the child should be age and gender appropriate. The pediatric dentists have to realize the potential of the child and then try to implement any behavior management technique for that particular child. In short, it has to be customized in accordance to the child in the present scenario. ${ }^{4}$ The present scenario has suddenly changed, with the world being affected by COVID-19.COVID-19, known as the Coronavirus Disease 19, is the viral infectious disease which rapidly developed as a pandemic. COVID-19 has an etiologic agent, i.e., severe acute respiratory syndrome coronavirus 2 (SARSCoV-2). SARS-CoV-2 was first discovered in 2019 in Wuhan, China. Then it unfortunately spread globally, resulting in the 2019-2020 pandemic, as declared by the World Health Organization (WHO). ${ }^{5}$ There are more than 342 millions affected and almost 10 million deaths worldwide. ${ }^{6}$ This virus has affected all the people showing no such predilection, but elderly people and immunocompromised people are affected more severely. Most of the countries are under lockdown for more than a month, some even more. All the offices, industries, and workplaces have been closed to maintain social distancing. The healthcare professionals, especially the dentists, are at the highest risk of coming in contact with this virus when working. ${ }^{7}$ But it has also to be seen that sooner or later the lockdowns will end, and the dentists will definitely have to start their consultations and work in a routine manner. But their routine will not be a normal routine anymore in this pandemic. The dentists have to take extra precautions, both for themselves as well as their patients and clinical staffs. The standard operating protocols have to be in place while working in the clinic so that the dentists are not infected from this virus. ${ }^{8}$ In this scenario, the child patients will be affected more, because of the changing surroundings and clinic environment.

\section{The impact of the current pandemic situation on children and their parents}

\section{The impact of COVID-19 on children}

The children have lesser immunity and weaker awareness of self-prevention and therefore should be the main focus during this pandemic. ${ }^{9}$ During any epidemic/pandemic, the psychological changes should be monitored for children in a proper manner. Previous studies state that children's psychological behavior is easily affected by their surroundings. ${ }^{10}$ At this point of time, children aren't able to attend schools and meet with their peers face-to-face. Such long-term negative events affect children and they tend to react negatively. Many haphazardness occurs such as increased use of electronic gadgets, reduced learning times, and changes in sleep patterns too. ${ }^{11}$ Apart from that, parents' stressful conditions during these times will also influence a child's behavior. ${ }^{12}$ A Child's bad behavior can impact their overall development. A single child will have less negative behavior than multiple children in a family setting as the children only have more awareness regarding the stress caused by surrounding environment. The pandemic situation in cities is relatively more serious than rural areas increasing the bad mood of parents which in turn can result in bad behavior in children. ${ }^{13}$ 


\section{The impact of COVID-19 on parents}

In the peak of the pandemic, parents also will have serious negative emotions which can include anxiety, depression, frustration, anger, and so on. ${ }^{14}$ The manifestations of these emotions may be revealed as excessive attention on the progression of pandemic, excessive attention to the health of themselves and their families and unreal suspicion of them being infected without any reason. In addition, parent's excessive attention on their child again will have an impact on the children's behaviour. ${ }^{15}$ Higher education levels of parents will mean that they will have deeper understanding of the situation, which can lead to psychological panic situations and these in turn again will affect the children at home. ${ }^{16}$

All these scenarios have to be taken into consideration in knowing what kind of behavior we are going to experience as a child enters the clinic along with the parents.

During the COVID-19 pandemic and together with all other medical activities, routine dental work in most countries was suspended and postponed in relation to the progress of the epidemic situation, with the recommendation of limiting dental interventions to emergency situations. Hence, there is the need to be able to follow the oral health status of children only through adequate remote interaction with parents via virtual meets, who must be properly trained on the recognition of diseases that do not require immediate intervention and which can therefore be managed with palliative measures, waiting to be treated in an outpatient setting at the end of the epidemic emergency. As far as possible, we, as pediatric dentists, should take up only emergency cases and try to defer elective cases like delays of deciduous teeth exfoliation, eruptive gingivitis and chronic periapical periodontitis which can be managed with antibiotics and analgesics. In case of emergencies, we have to advise the parents to get the child to the clinic; but we also have to make them aware of the changes in the clinic and the standard operating protocols in this current pandemic situation.

\section{The changed clinic environment and child behavior management}

Behavior management is the means by which the dental health team effectively and efficiently provides treatment and thus building a positive dental attitude. ${ }^{17}$ The pediatric dentists are in a position to provide treatment to the children, cooperative or uncooperative with the behavior management techniques available both pharmacologically and non-pharmacologically. The present situation of COVID-19 has bound the pediatric dentists to think their behavior management strategies in a different way. The COVID-19 not only seriously threatens the physical and mental health of patients and their parents but also effects the mental health of the public. ${ }^{18}$ The methods used prior to this pandemic will have to be modified and also newer methods have to be adopted. Here in this research, we will outline few situations which will change in a pediatric dental clinic setup and will also discuss how we can manage the anxieties and fears in children.

\section{The pediatric dental clinic setup}

The pediatric dental clinics are used to having lot of attractions which are child-specific and also for the parents to be kept engaged. ${ }^{19}$

\section{The reception area}

The reception section is the first space that the patients enter, so it is of great importance. The patients (and their parents too) judge and view details of the entire clinic the moment they enter the reception area. The design of this space such that it attracts the children and the parents. Now with the changed scenario due to the pandemic, the reception will have a changed look for the child as he/she enters the clinic. This is because the child has to undergo temperature checks with infrared thermometer, use of pulse oximeter for oxygen saturation as children are mostly asymptomatic, use of sanitizers, use of shoe covers along with the attire of the receptionist, who will be mostly covered up in a PPE. This itself will be an overwhelming situation for a child dental patient. The receptions will not have many decorative pieces to attract children as it can again lead to virus being settled on them. ${ }^{20}$ 


\section{What can be done to reduce fears?}

Dental practitioner should always talk on phone and have a proper telephone triage with parents or guardians to obtain all the possible information about the child patient's health status as well as oral symptoms. Being informed will make one able to understand if dental procedure represents an urgent need or it can be postponed without any harm. Also the parents have to be informed that they have to prepare their children for the situation. They can show them various videos in which the healthcare professionals have worn PPEs and are using infrared thermometers. Hence pre-appointment calls and letters will be an effective behavior management tool which will aid in reducing fear in a child. To make sure that no more than one pediatric patient and an accompanying person should wait in the preoperative room at a time, proper dental emergency appointments must be organized.

\section{The waiting area}

The waiting areas are designed in such a way that it will keep both the children and the parents engaged while they wait for their turn. The waiting area has number of magazines for parents, comics and even color books for children. Some clinics also have aquariums to keep the children entertained. Here both the children and parents wait for their turn inside the operatory. Unfortunately the waiting area will not have these in the recent pandemic scenario. Previously the waiting areas were so lively that children became friends with other children. Simultaneously, their fears were reduced too. But now, when social distancing is the most important norm, child patients will not be allowed by their parents to mingle with other children as they will be worried about the transmission of this virus. ${ }^{21}$

\section{What can be done?}

This pandemic has induced bad habits when it increased almost every child's screen time. Most children are glued to televisions or smart phones. Hence, all the clinics must provide free Wi-Fi so that the children could be kept engaged while patiently waiting for their turns to come. Here the dentists can utilize some useful tricks. The television set can be made to show treatment being done on a child who is cooperative, so that the children know what to expect inside. Here the audiovisual aids will be helpful for behavior management. Another important thing to be kept in minds is to keep the waiting time as short as possible. This is again keeping in mind that attention span of children is short as well as in these pandemic times they are already irritated staying at home. ${ }^{22}$

\section{The play area}

The play area is an integral part in any pediatric dental clinic and an added attraction for child patients. Larger clinics (larger areas) having a lot of space use to keep lot of play items for children, e.g., slides, gaming consoles, items for paintings etc. Even for smaller clinics this was normal to keep play items in play room. ${ }^{23}$ But again this is bound to change with socialdistancing norms and to reduce the transmission of coronavirus very few items can be kept in this area. All the items have to be cleaned properly every time a child touches it so most clinics will do away with lot of play items. It must always be kept in mind that the toys provided for children may be a potential source of drastic crossinfection. It is observed that soft toys are most contaminated among all, are difficult to disinfect, and may re-contaminate rapidly when compared to toys that are hard in nature. Then there is the fear of virus settling on these items if many children play with these items; and mostly we now know that children may be asymptomatic carriers of this deadly virus. ${ }^{24}$

\section{What can be done?}

Always allow only one child at a time inside the play area. Be careful in sanitizing the area as soon as the child comes out and wait for some time (at least 30 minutes).Then you can allow another child to go inside the play area.

\section{The treatment room}

This is the room where the child will have a lot of fear about. The dental chair in the treatment room is a device in which the patient undergoes treatment while at the same time tolerates dental 
panic and anxiety. A dental unit with a variety of instruments, such as the turbine, handpieces, suction, lamp, etc. may be extremely fearpromoting for the children, especially for those who are going to the dental office for the very first time. This room is one of the most significant parts of the clinic for a pediatric dental clinic. This room is a place where the child should not get anxious and stressed when arriving.

Therefore, it must be designed and made in such a way that the child feels comfortable. The atmosphere inside the treatment room should be cheerful and child-friendly. Dental units with pediatric designs should be replaced by the conventional units. For the distracting the children, music could be played with the help of headphones or television can be set with cartoon serials. $^{24}$

But now with this pandemic scenario, the dentist and the dental assistant will be wearing PPEs along with face shields which can be overwhelming for the child on a first visit. The attire itself may scare the child immensely. The pediatric dentists will more often be using conventional designs of dental chair as pediatric designs might not allow them to work free with all standard protocols in place. The basic behavior management techniques of communication, tellshow-do assumes an important role in this scenario.

The pharmacological means of behavior management, i.e., nitrous oxide sedation may not be feasible in these times as they will again increase the work load on the dentist and the assistant for disinfecting the entire unit. If aerosol generating procedures (AGP) are to be done, the use of high evacuation suction is a must which again can promote fear in the child. There are suggestions to minimize the use of these tools; if this is not possible, the last appointment of the day should be intended for those patients who need dental treatments requiring the use of highspeed rotating instruments. ${ }^{25}$ The parent may not be allowed inside the operatory to maintain social distancing and keeping in mind that all patients might be carriers of this virus. The situation might become daunting for the child and fear may increase to a greater extent. ${ }^{26}$

\section{What can be done?}

The most fear-promoting thing inside the operatory will be the fear from unknown as the child would have sensed the changes in the clinic setup the moment he walked in. Here it is the parents' responsibility to reassure the child about the environment. The next challenge for the child to face would be the pediatric dentist and the assistant's attires as they will be in PPEs. The PEs can be made little child friendly. The face masks can have the designs of a cartoon mouth; the face shields can also incorporate interesting designs to make them less fearful for the patient. The child has to be explained each procedure slowly. The communication (verbal and nonverbal) has to be minimized in this pandemic. It is advisable for the pediatric dentists to record and show all the procedures to the child patients on the television which can be placed inside the operatory. The parents should be allowed inside the operatory irrespective of the age of the child as most children will be emotionally unstable in these testing times where they are not allowed to play with their friends. The parents also should follow the standard operating protocol of the clinics. The parents should be given the option of general anesthesia in hospital setups if the child does not cooperate. The use of sedation in this pandemic in clinics is not much advisable. The children lacking cooperative ability can be managed with physical restraints. Furthermore, restraining devices used to control movements of pediatric patients such as Velcro Fasteners may also be contaminated and should be disinfected accordingly. 27 Thepediatric dentists also have to treat the special children under general anesthesia in hospital care with prior COVID-19 tests. Furthermore, pediatric dentists must take up all the responsibilities of safeguarding children with compromised systemic health no matter what, as these child patients areaways at greater risk of complications that may arise from any dental infection and specially-abled children (e.g., autistic pediatric patients). This is because many a times such patients' behavior may become impossible to control properly in case of severe dental pain. ${ }^{28}$

\section{Discussion and Conclusions}

COVID-19 has now spread throughout the world like wildfire. While there is currently no 
vaccine to prevent COVID-19, doctors and researchers are learning more about it every day. Symptoms of COVID-19 range from mild to severe. These generally begin 2-14 days after being exposed to the virus and often include coughing and shortness of breath. At least two of these symptoms can also suggest a person has COVID-19 such as follows: fever, chills, repeated shaking with chills, muscle pain, headache, sore throat, and a new loss of taste or smell. ${ }^{29}$ Early research suggests that fewer children than adults with COVID-19 get a fever, coughing, or shortness of breath. It is also observed that some children attacked with COVID-19 had to be hospitalized as well. However, severe illness has been reported in children, most often in infants less than a year. ${ }^{30}$ In the recent times, the outbreak of the coronavirus in dentistry is one of the greatest challenges that the many of the parents are facing. The pandemic caused due to Coronavirus seems to have a major impact on the parents. If there is a need for the emergency dental treatment, they prefer to stay at home and use a home remedy or give pain killers to relieve children's dental pain instead of referring to dental offices or clinics. ${ }^{31}$ However, with the application of standard precautions and protocols consistently; there is not much to be afraid of. Before the pediatric dentist can start practicing, they should be aware of the local guidelines of their states and countries. Healthcare updates are changing at a rapid pace, and it's really confusing and alarming to start practice normally. The dentists and the attendants in the clinic should be aware of standard operating protocols. Initially the preference should be given to those children who have dental emergencies. The next preference is to be given for preventive protocols like pit and fissure sealants, silver diamine fluoride application, fluorides, Hall Technique, atraumatic restorative treatment. ${ }^{32,33,34}$ All these are nonAGP. The role of parents during these times for behavior management will be more as they are spending more time with their children; thus they can understand the psychology of the child better than the pediatric dentists. The pediatric dentists now have to be more dependent on technology. The first consultation and communication with the child must be made virtually so that the child becomes acquainted with the pediatric dentist.
Here the pediatric dentist can give them oral hygiene ,hand hygiene, sneeze etiquettes, and also tell them a little about this viral disease including the changes he/she will witness in the clinic. All these have to re-enforced by the parents until the time arrives for visiting the clinic again. The pandemic has and will continue to change the way pediatric dentists practice. It becomes essential that in these testing times, we do not leave our little patients to suffer. The ongoing COVID-19 spread is a public health emergency and global threat. Governments all around the world have ordered citizens to stay at home as an emergency measure and implemented school closures to prevent further spread of the infection. More than 250 million children and adolescents in 165 countries are affected by the closures. ${ }^{35}$ Under such situations, physical and mental health problems are significant concerns. Particularly, children and adolescents' lifestyle behaviors, such as physical activity and sedentary behavior, may have been drastically impacted due to the prolonged school closures and home confinement during the COVID-19 pandemic. ${ }^{36}$ This has led to an increased negative behavior being observed in children trapped in their homes. Pediatric dentists have to keep in view the situation and manage the children accordingly. ${ }^{37}$

The COVID-19 has changed the way we used to practice with our little patients; but we, as pediatric dentists, have to overcome the barriers and give the best possible treatment to them while maintaining all the protocols. Till now no published data has given any importance to the behavior and the behavior management techniques to be followed by pediatric dentists.

The pediatric dentists should keep in mind the complete mental picture of the child before undertaking any treatment in this pandemic. As mentioned previously, preventive measures against COVID-19 in dental practice mostly include clinical or telephonic triage supported by an elaborate questionnaire on the present movements and symptoms, oral rinses with $1 \%$ hydrogen peroxide, body temperature measurement, and the use of specific PPEs. ${ }^{38,39} \mathrm{It}$ must be made sure that preventive measures are properly employed for child patients as much as possible. Along with parents and dental staffs in the clinic, the behavioral changes which have 
been identified in children have to be managed with appropriate management skills. In this process, the parents can be of great help. Moreover, they have to be informed over telephone. Some points are as follows: ${ }^{40,41}$

1) They must be creative in their home-based fun activities. They must know how to encourage and support their children to be active in innovative but safe ways. Suggestions include trying new leisure hobbies, co-participation in activities, using online physical and health activity apps, and, last but not least, getting outdoors more frequently (while maintaining proper public safety advises).

2) Parents must be responsible in setting routines for their children, including regular sleeping and waking times, supervised time for screens, and proper time for quality family moments.

Children facing unexpected events usually exhibit various stress reactions, as confirmed in the study performed in China during the COVID19 epidemic. ${ }^{42}$ Resilience, the personal attributes that help children manage everything from little disappointments to big life traumas, should be nurtured and implemented by public health programs in children. This should be properly supported by healthcare personnel, families, and friends - so that they can easily overcome these stresses.

In helping the child psychologically, these small but effective approaches definitely come in handy. It will be much easier for us to manage the children in the clinics once they are emotionally strong enough to understand the present scenario.

\section{REFERENCES}

1. American Academy of Pediatric Dentistry. Policy on medically necessary care. Pediatr Dent 2015;37:18-22

2. Baier K, Milgrom P, Russell S, Mancl L, Yoshida T. Children's fear and behavior in private pediatric dentistry practices. Pediatr Dent 2004;26:316-321

3. Wu L, Gao X. Children's dental fear and anxiety: exploring family related factors. BMC Oral Health 2018;18:100-107

4. Brill WA. The effect of restorative treatment on children's behavior at the first recall visit in a private pediatric dental practice. J Clin Pediatr Dent 2002; 26:389-394

5. World Health Organization. Coronavirus disease (covid-19) pandemic [Internet] [cited 20 Oct 2020]. Avaliable from: https://www.who.int/news-room/detail/30-012020-statement-on-the-second-meeting-of-theinternational-health-regulations-(2005)rmergency-committee-regarding-the-outbreak-ofnovel-coronavirus-(2019-ncov).

6. The workers who face the greatest coronavirus risk. The New York Times (New York) [Internet] [cited 10 Oct 2020]. Available from: https://www.nytimes.com/interactive/2020/03/ 15 /business/economy/coronavirus-workerrisk.html

7. Coronavirus disease [Internet] [cited 10 Oct 2020]. Available from: https://www.worldometers.info/coronavirus/cor onavirus-death-toll.

8. Moodley R, Naidoo S, Wyk J V. The prevalence of occupational health related problems in dentistry: A review of the literature. J Occup Health 2018;60:111-125

9. Fang F. and Luo X. Facing a major epidemic of 2019 novel corona virus infection: pediatricians' thoughts. Chinese J Pediatr 2020;58:81-88

10. Gao X., XingS, JhaoJ. Parental psychological control and the relationship with children's psychosocial functioning. Advances in Psych Scien 2016;24:1792-1801

11. Buchanan H. Assessing dental anxiety in children: The revised smiley faces program. Child Care Health Dev 2010;36:534-538.

12. Jin Z, Zhang X, Han ZR. Parental Emotion Socialization and Child Psychological Adjustment among Chinese Urban Families: Mediation through Child Emotion Regulation and Moderation through Dyadic Collaboration. Front Psychol 2017;8:2198-2204

13. Li Y, Liu L, Lv Y, Xu L, Wang Y, Huntsinger CS. Mother-child and teacher-child relationships and their influences on Chinese only and nononly children's early social behaviors: The moderator role of urban-rural status. Child Youth Serv Rev 2015;51:108-116

14. Demby K.P, Riggs S.A, Kaminski PL. Attachment and family processes in children's psychological adjustment in middle childhood. Fam Process 2017;56:234-249 
15. Kaseva K, Dobewall H, Yang X, PulkkiRåback L, Lipsanen J, Hintsa T.et.al. Physical Activity, Sleep, and Symptoms of Depression in Adults - Testing for Mediation. Med Sci Sports Exerc 2019;51:1162-1168.

16. White, S. H. Some educated guesses about cognitive development in the pre-school years. In: R.D Hess and R M Bear, editors. Early education: Current theory, research, and action. Chicago: Aldine, Press; 2017.p.203-214

17. American Dental Association Commission on Dental Accreditation. Accreditation Standards for Advanced Specialty Education Programs in Pediatric Dentistry. $1^{\text {st }}$ ed. Chicago.: American Dental Association; 2013

18. Park JG. Color perception in pediatric patient room design: Healthy children vs. pediatric patients. High Educ Res Dev 2009;2:6-28

19. Nuttall NM, Bradnock G, White D, Morris J, Nunn J. Dental attendance in 1998 and implications for the future. $\mathrm{Br}$ Dent $\mathrm{J}$ 2001;15:357-3587

20. Mittal R, Sharma M. Assessment of psychological effects of dental treatment on children. Contemp Clin Dent 2012;3:2-7

21. Gustafsson A, Arnrup K, Broberg AG, Bodin L, Berggren U. Psychosocial concomitants to dental fear and behaviour management problems. Int J Paediatr Dent 2007;17:449-459

22. Gaertner BM, Spinrad TL, Eisenberg N. Focused Attention in Toddlers: Measurement, Stability, and Relations to Negative Emotion and Parenting. Infant Child Dev 2008;17:339-363.

23. Nanda U, Chanaud CM, Brown L, Hart R, Hathorn K. Pediatric art preferences: Countering the "one-size-fits-all" approach. High Educ Res Dev 2009;2:46-61

24. Izzetti R, Nisi M, Gabriele M, Graziani F. COVID-19 transmission in dental practice: brief review of preventive measures in Italy. J Dent Res 2020;99:1030-1038

25. Peng X., Xu X., Li Y., Cheng L., Zhou X., Ren B. Transmission routes of $2019-\mathrm{nCoV}$ and controls in dental practice. Int $\mathrm{J}$ Oral Sci 2020;12:1-6

26. Wells MH, McCarthy BA, Tseng CH, Law CS. Usage of behavior guidance techniques differs by provider and practice characteristics. Pediatr Dent 2018;40:201-208.
27. Carmona JR. COVID-19 Outbreak and Dentistry. Odovtos-International J Dent Sci 2020; 22:9-15

28. Royal College of Surgeons of England Recommendations for Paediatric Dentistry during COVID-19 pandemic. [Internet] [cited 28 Aug 2020]. Available from: https://www.rcseng.ac.uk/dentalfaculties/fds/coronavirus/

29. Meng L, Hua F, Bian Z. Coronavirus disease 2019 (Covid-19): Emerging and future challenges for dental and oral medicine. J Dent Res 2020;99:481-487

30. Lauer SA, Grantz KH, Bi Q, Forrest KJ, Zheng Q, Hannah RM et al. The Incubation Period of Coronavirus Disease 2019 (COVID-19) From Publicly Reported Confirmed Cases: Estimation and Application. Ann Intern Med 2020;172:577-582

31. Dong Y, Mo X, Hu Y, et al. Epidemiological characteristics of 2143 pediatric patients with 2019 coronavirus disease in China. Pediatrics 2020:e20200702

32. Lu X, Zhang L, Du H, Zhang J, Li YY, Qu J, et al. SARS-CoV-2 Infection in Children. N Engl J Med 2020;382:1663-1665

33. De Amorim RG, Frencken JE, Raggio DP, Chen X, Hu X, Leal SC. Survival percentages of atraumatic restorative treatment (ART) restorations and sealants in posterior teeth: an updated systematic review and meta-analysis. Clinical Oral Invest 2018; 22: 2703-2725

34. Schwendicke F, JägerAM, Paris S, Hsu LY, Tu YK. Treating pit and fissure caries: a systematic review and network meta-analysis. J Dent Res 2015;94:522-533

35. Seifo N, Cassie H, Radford JR, Innes NP. Silver diamine fluoride for managing carious lesions: an umbrella review. BMC Oral Health 2019;19:145-155

36. Innes NP, Evans DJ, Stirrups DR. Sealing caries in primary molars: randomized control trial, 5-year results. J Dent Res 2011;90:1405-1410

37. Xiang M, Zhang Z, Kuwahara K. Impact of COVID-19 pandemic on children and adolescents' lifestyle behavior larger than expected. Prog Cardiovasc Dis 2020;20:3009630097. 
38. Ma Q.X., Shan H., Zhang H.L., Li G.M., Yang R.M., Chen G.M. Potential utilities of mask wearing and instant hand hygiene for fighting SARS-CoV-2. J Med Virol 2020:92:1562-1271

39.Lo GR. The Severe Acute Respiratory Syndrome Coronavirus-2 (SARS COV-2) in dentistry. Management of biological risk in dental practice. Int $\mathrm{J}$ Environ Res Public Health 2020;17:3067-3072

40. Moore, SA, Faulkner G, Rhodes RE, Brussoni $\mathrm{M}$, Tala CB, Ferguson LJ, et al. Impact of the COVID-19 virus outbreak on movement and play behaviours of Canadian children and youth: a national survey. Int $\mathrm{J}$ Behav Nutr Phys Act 2020:17;1-11

41. Halla G., Laddu D.R., Phillips S.A., Lavie C.J., Arena R. A tale of two pandemics: how will COVID-19 and global trends in physical inactivity and sedentary behavior affect one another? Prog Cardiovasc Dis 2020 Apr 8. Doi: 10.1016/j.pcad.2020.04.005

42. Jiao WY, Wang LN, Liu J, Fang SF, Jiao FY, Massimo PM et al. Behavioral and Emotional Disorders in Children during the COVID-19 Epidemic. J Pediatr 2020;221: 264-266

\section{How to cite this article:}

Sonu Acharya. Behavior management in Pediatric Dentistry during and after Corona pandemic. Contemp Pediatr Dent 2020:1(1):13-21.

\section{Declarations}

Acknowledgemetns: Not applicable.

Conflict of Interest Statement: The author discloses no potential conflicts of interest.

Ethics Statement: This study does not require approval from the ethics committee.

Informed Consent: Not required.

Author contributions: Conception and design: $S A$; Acquisition of data: $S A$; Interpretation of data: $S A$; Drafting article: $S A$; Revision artice: $S A$; Final approval: $S A$

Funding: This work is not finantiated.

Data Availability: The data used to support the findings of this study can be made available upon request to the corresponding author.

Peer-review: Externally double-blinded peer-reviewed. 